\title{
Características de óbitos dos pacientes internados em uma unidade de terapia intensiva de hospital terciário
}

\section{Characteristics of deaths of hospitalized patients in Intensive Care Unit of a tertiary hospital}

\author{
Amanda Melo Vieira ${ }^{1,}$ (D), Ezequiel Aguiar Parente ${ }^{1} \mathbb{D}$, Lean de Sousa Oliveira ${ }^{1} \mathbb{D}$, Anaiara Lucena Queiroz ${ }^{2,3}$ (D), Iara Serra Azul \\ Machado Bezerra $^{3}$ (D) , Hermano Alexandre Lima Rocha² (D)
}

1. Discente do Curso de Medicina do Centro Universitário Christus (UNICHRISTUS), Fortaleza, CE, Brasil. 2. Docente do Curso de Medicina do Centro Universitário Christus (UNICHRISTUS), Fortaleza, CE, Brasil. 3. Médica do Hospital Geral de Fortaleza (HGF), Fortaleza, CE, Brasil.

\begin{abstract}
Resumo
Introdução: Estudos comparam taxa de óbitos de Unidade de Terapia Intensiva (UTI) no Brasil que se encontra acima da internacional, não se sabendo se isso é devido ao sistema público brasileiro ou a falhas de registro. Objetivo: Traçar uma relação entre mortalidade prevista e características dos pacientes internados na UTI de hospital terciário de grande porte do município de Fortaleza, Ceará. Métodos: Trata-se de um estudo retrospectivo, analítico e transversal sobre pacientes internados na UTI do Hospital Geral de Fortaleza de janeiro a novembro de 2016. Resultados: No período da pesquisa, foram estudados 137 pacientes; desse total vieram a óbito 31 pacientes (24,48\%). De acordo com a prioridade estabelecida pela Society Critical Care Medicine para Unidade de Terapia Intensiva, tivemos $53,3 \%$ na prioridade I, $10,0 \%$ na prioridade II, $30,0 \%$ na prioridade III, 3,3\% na prioridade IVA e 3,3\% na prioridade IVB, resultados estatisticamente significantes $(\mathrm{p}=0,012)$. Constatou-se neste trabalho que $80,6 \%$ dos óbitos haviam desenvolvido sepse durante o internamento na UTI $(\mathrm{p}<0,001)$. Aplicando-se os escores de mortalidade APACHE-II e SOFA, observa-se que o APACHE-II com escore médio de 22 pontos e com mortalidade prevista média de 55,7 ( $\mathrm{p}<0,001$ ), enquanto, o SOFA teve média no primeiro dia de 7 pontos ( $\mathrm{p}<0,001$ ), no quinto dia de 5 pontos $(p=0,004)$ e no décimo dia de 5 pontos $(p=0,004)$. Conclusão: Observou-se a importância da análise epidemiológica em relação aos óbitos ocorridos em Unidades de Terapia Intensiva, visando demonstrar a necessidade de se conhecer melhor a aplicabilidade dos escores SOFA e APACHE II e as medidas que devem ser adotadas.
\end{abstract}

Palavras-chave: Mortalidade. Escores de Disfunção Orgânica. Unidades de Terapia Intensiva. APACHE. SOFA.

\begin{abstract}
Introduction: Studies comparing rate of deaths of an Intensive Care Unit (ICU) in Brazil that is above international standards, not knowing whether this is due to the Brazilian public system or failures of record. Objective: To establish a relationship between predicted mortality and characteristics of patients admitted to the Intensive Care Unit of a hospital in city of Fortaleza, Ceará. Methods: This was a retrospective study, analytical and cross patients admitted to the Intensive Care Unit of a General Hospital of Fortaleza during the period from January to November 2016. Results: At the time of the study, were studied 137 patients, this total of patients died 31 patients (24.48\%). In accordance with the priority set by the SCCM to ICU, we had $53.3 \%$ in the priority I, $10.0 \%$ in the priority II, $30.0 \%$ in the priority III, 3.3\% VAT priority and 3.3\% in the priority IVB, the results were statistically significant (p=0.012). It was found in this study that $80.6 \%$ of deaths developed sepsis during hospitalization in the ICU $(\mathrm{p}<0.001)$. Applying the mortality scores APACHE-II and SOFA, it is observed that the apache-II with a mean score of 22 points, with a standard deviation of 8 points, and with the predicted mortality average of $55.7(\mathrm{p}<0.001)$. While, the SOFA was on the first day of 7 points $(\mathrm{p}<0.001)$, on the fifth day of 5 points $(\mathrm{p}=0.004)$ and on the tenth day of 5 points $(\mathrm{p}=0.004)$. Conclusion: There was the importance of epidemiological analysis in relation to deaths occurring in Intensive Care Units, aiming to demonstrate the necessity of knowing more about the applicability of the scores SOFA and APACHE II and the measures that ought to be taken in cases of high mortality risk.
\end{abstract}

Key words: Mortality. Scores of organic dysfunction. Intensive Care Units. APACHE. SOFA.

INTRODUÇÃO

O envelhecimento populacional com rápido crescimento da globalização e tecnologia, trouxe também a transição epidemiológica para o Brasil, observando-se o predomínio de doenças crônicas degenerativas sobre as doenças infectoparasitárias ${ }^{1}$. Devido a isso, o Sistema Único de Saúde (SUS) adaptou-se ativamente, por meio de investimentos nos hospitais públicos nacionais, para melhorar a abordagem dessas doenças com a criação das Unidades de Terapias Intensivas, por exemplo².

A Unidade de Terapia Intensiva (UTI) é uma unidade hospitalar de alta complexidade voltada para pacientes críticos com a possibilidade de reversibilidade do quadro clínico, que dispõe de uma equipe multiprofissional, observação médica, equipamentos e monitorização contínua ${ }^{3,4}$.

Estudo canadense mostrou que aproximadamente $0,5 \%$ da população adulta será internada em UTIs, apresentando uma maior incidência em homens idosos com menor renda familiar ${ }^{5}$. Na maioria das UTIs, utiliza-se a gravidade das doenças para prever a mortalidade desses pacientes ${ }^{6}$. Observa-se elevada taxa de mortalidade nesse ambiente, variando de acordo com os artigos internacionais entre 5,4 e $33 \% \%^{7,8,9,10}$. Porém, na realidade brasileira, encontra-se uma taxa de óbitos acima

Correspondência: Amanda Melo Vieira. Rua João Adolfo Gurgel, 133, bairro Cocó, CEP: 60192-345, Fortaleza, Ceará, Brasil. E-mail: amandamelovieira@ gmail.com

Conflito de interesse: Não há conflito de interesse por parte de qualquer um dos autores.

Recebido em: 8 Fev 2018; Revisado em: 26 Set 2018; 27 Out 2018 Aceito em: 11 Nov 2018 
da internacional, variando de 36,9 a $50,74 \%$, não se sabendo se isso é devido ao sistema público brasileiro ou a falhas de registro $^{11,12,13}$.

Esse artigo tem como objetivo traçar uma relação entre a taxa de mortalidade e as características dos pacientes internados na Unidade de Terapia Intensiva (UTI) de um hospital terciário de grande porte do município de Fortaleza/CE, por meio dos escores SOFA e APACHE-II.

\section{MÉTODOS}

\section{Desenho do estudo}

Trata-se de um estudo retrospectivo, analítico e transversal feito por meio de prontuários médicos dos pacientes internados na unidade no período de janeiro a novembro de 2016.

\section{Local e período do estudo}

O estudo foi desenvolvido na Unidade de Terapia Intensiva do Hospital Geral de Fortaleza, na cidade de Fortaleza - CE no período de agosto de 2016 a julho de 2017. O Hospital Geral de Fortaleza (HGF) é um hospital que pertence à rede do Sistema Único de Saúde (SUS), considerado o maior hospital da rede estadual do Ceará e referência em procedimentos de alta complexidade. Atualmente, dispõe de 563 leitos, divididos entre eletivo, emergência, obstetrícia e unidade de terapia intensiva (UTI) adulta e neonatal. Sua UTI disponibiliza à rede estadual 39 leitos de alta complexidade.

\section{População e amostra}

A população foi constituída com os pacientes internados na UTI do HGF. A composição da amostra foi de 147 pacientes internados no período descrito acima. Para essa amostra, concede-se, para um nível de significância de 0,05 , um erro amostral de $9 \%$.

\section{Critérios de inclusão e exclusão}

Os pacientes incluídos no estudo foram selecionados de forma aleatória, os quais haviam sido admitidos na Unidade de Terapia Intensiva (UTI) do HGF durante o período estudado e com idade maior que 14 anos. Não houve critérios de exclusão.

Instrumentos para a coleta de dados

As variáveis estudadas foram a idade, a procedência do paciente, o(s) diagnóstico(s), os escores do APACHE e do SOFA, a existência de comorbidades (hipertensão arterial sistêmica, diabetes, doença arterial coronariana, insuficiência cardíaca, doença respiratória crônica, tabagismo, etilismo, cirrose hepática, entre outras), a existência de disfunções orgânicas e suportes usados na admissão (drogas vasoativas, inotrópicos, ventilação mecânica, entre outros), a presença, ou não, de sepse e os dados da evolução do paciente na UTI.
O escore SOFA é calculado por meio da soma das disfunções de seis sistemas orgânicos: renal, neurológico, respiratório, cardiocirculatório, hepático e hematológico. Cada disfunção é graduada de 0 a 4 pelo escore SOFA; portanto, a soma pode variar de 0 até 24 . O escore APACHE II é calculado após as primeiras 24 horas do paciente no ambiente de terapia intensiva por meio de 12 variáveis fisiológicas e laboratoriais. Por meio dessa pontuação aliada à etiologia da patologia de base, um software calcula ainda a mortalidade prevista e individualizada para cada paciente.

\section{Análise dos dados e apresentação dos resultados}

Os dados foram inicialmente alimentados no software Excel, versão 2013, Microsoft Inc ${ }^{\circledR}$. Em seguida, forma realizadas análises descritivas, com cálculo de frequência e percentuais ou médias, medianas, desvios padrões e máximos ou mínimos, conforme adequado. A normalidade das variáveis foi testada com o teste de Kolmogorov-Smirnov. Foram utilizados testes de qui-quadrado para variáveis categóricas e t de student ou MannWhitney conforme adequado de acordo com a normalidade. Foi utilizado o software SPSS v 23 for Windows, IBM Inc ${ }^{\circledR}$ para analisar os dados.

\section{Aspectos éticos}

A pesquisa foi devidamente submetida e aprovada pelo Comitê de Ética do Centro Universitário Christus, respeitando todos os princípios éticos que regem a resolução 466/12 do Conselho Nacional de Saúde - CSN/Ministério da Saúde - MS, que regulamenta a pesquisa em seres humanos. $O$ projeto foi registrado com o número CAAE 55700516.8.0000.5049 na plataforma Brasil.

\section{RESULTADOS}

No período da pesquisa, foram estudados 137 pacientes. Desse total, vieram a óbito 31 (24,48\%). Apresenta-se um discreto predomínio do sexo feminino $(51,6 \%)$. A média da idade dos que evoluíram a óbito foi de 60 anos com um desvio-padrão de 16 anos $(p=0,004)$. Houve um predomínio dos pacientes provenientes da Região Metropolitana de Fortaleza (RMF) no percentual de óbitos $(67,7 \%)$.

Prevaleceu, também, a mortalidade em mais de 48 horas $(83,9 \%)$, versus $16,1 \%$ antes 48 horas do internamento. 0 período de internamento médio até o óbito foi de 20 dias com desvio padrão de 18 dias. (Tabela 1)

Houve o predomínio de óbitos relacionados a casos clínicos $(74,2 \%)$, seguidos de casos cirúrgicos de emergência $(19,4 \%)$ e casos cirúrgicos $(6,5 \%)$.

De acordo com a prioridade estabelecida pela SCCM para UTI, tivemos $53,3 \%$ na prioridade I, $10,0 \%$ na prioridade II, 30,0\% na prioridade III, 3,3\% na prioridade IVA e $3,3 \%$ na prioridade IVB, resultados estatisticamente significantes $(p=0,012)$. 
Tabela 1. Características demográficas dos pacientes internados na Unidade de Terapia Intensiva (UTI) do Hospital Geral de Fortaleza

\begin{tabular}{|c|c|c|c|}
\hline \multirow[b]{2}{*}{ Características } & \multicolumn{2}{|c|}{ Média DP) ou N (\%) } & \multirow{2}{*}{$\begin{array}{l}\text { Valo } \\
\text { do }\end{array}$} \\
\hline & $\begin{array}{c}\text { Não evoluiu a } \\
\text { óbito }\end{array}$ & óbito & \\
\hline Idade & $48 \pm 20$ anos & $60 \pm 16$ anos & $<0,05$ \\
\hline \multicolumn{4}{|l|}{ Gênero } \\
\hline Masculino & $59(55,7 \%)$ & $15(48,4 \%)$ & 0,475 \\
\hline Feminino & $47(44,3 \%)$ & $16(51,6 \%)$ & \\
\hline \multicolumn{4}{|l|}{$\begin{array}{l}\text { Localização de } \\
\text { moradia }\end{array}$} \\
\hline RMF & $52(49,1 \%)$ & $21(67,7 \%)$ & 0,807 \\
\hline Outros & $54(50,9 \%)$ & $10(32,3 \%)$ & \\
\hline $\begin{array}{l}\text { Evolução ao } \\
\text { óbito }\end{array}$ & 106 & 31 & 0,086 \\
\hline $\begin{array}{l}\text { Tempo de } \\
\text { internamento }\end{array}$ & 190 dias & 110 dias & 0,266 \\
\hline
\end{tabular}

Colocando em foco um dos principais conflitos que os hospitais públicos enfrentam atualmente, devido ao número de leitos restritos, foi visto como resultado, neste estudo, que 9,7\% dos óbitos tinham admissão na UTI proveniente de mandados judiciais.

Os principais diagnósticos dos pacientes internados na UTI foram: Acidente Vascular Cerebral $(22,6 \%)$, pós-cirúrgicos $(19,4 \%)$, outras doenças neurológicas $(16,1 \%)$, infecções $(9,7 \%)$, insuficiência hepática $(9,7 \%)$, neoplasias $(9,7 \%)$, pancreatite $(6,5 \%)$, coma $(3,2 \%)$ e intoxicação $(3,2 \%)$. (Tabela 2 )

Tabela 2. Diagnósticos e suas prioridades de acordo com SCCM dos pacientes internados na Unidade de Terapia Intensiva (UTI) do Hospital Geral de Fortaleza

\begin{tabular}{|c|c|c|c|}
\hline \multirow[b]{2}{*}{ Características } & \multicolumn{2}{|c|}{ Média DP ou N(\%) } & \multirow[t]{2}{*}{ Valor do $p$} \\
\hline & Não evoluiu a óbito & Óbito & \\
\hline $\begin{array}{l}\text { Prioridades } \\
\text { (SCCM) }\end{array}$ & & & 0,012 \\
\hline I & $41(39,4 \%)$ & $4(12,9 \%)$ & \\
\hline II & $40(38,5 \%)$ & $16(53,3 \%)$ & \\
\hline III & $17(16,3 \%)$ & $3(10,0 \%)$ & \\
\hline IV-A & $6(5,8 \%)$ & $1(3,3 \%)$ & \\
\hline IV-B & $0(0 \%)$ & $1(3,3 \%)$ & \\
\hline Diagnósticos & & & 0,322 \\
\hline AVC & $28(26,4 \%)$ & $7(22,6 \%)$ & \\
\hline Coma & $10(9,4 \%)$ & $1(3,2 \%)$ & \\
\hline Infecção & $13(12,3 \%)$ & $3(9,7 \%)$ & \\
\hline
\end{tabular}

\begin{tabular}{|c|c|c|c|}
\hline \multirow[b]{2}{*}{ Características } & \multicolumn{2}{|c|}{ Média DP ou N(\%) } & \multirow[t]{2}{*}{ Valor do $p$} \\
\hline & Não evoluiu a óbito & Óbito & \\
\hline $\begin{array}{l}\text { Insuficiência } \\
\text { hepática }\end{array}$ & $2(1,9 \%)$ & $3(9,7 \%)$ & \\
\hline Intoxicação & $1(0,9 \%)$ & $1(3,2 \%)$ & \\
\hline Neoplasia & $10(9,4 \%)$ & $3(9,7 \%)$ & \\
\hline Outras & $5(4,7 \%)$ & $0(0 \%)$ & \\
\hline $\begin{array}{l}\text { Doenças } \\
\text { neurológicas }\end{array}$ & $7(6,6 \%)$ & $5(16,1 \%)$ & \\
\hline Pancreatite & $6(5,7 \%)$ & $2(6,5 \%)$ & \\
\hline Pós-cirúrgico & $24(22,6 \%)$ & $6(19,4 \%)$ & \\
\hline
\end{tabular}

Constatou-se, neste trabalho, que $80,6 \%$ dos óbitos desenvolveram sepse durante 0 internamento na UTI $(p<0,001)$. De acordo com a gravidade da sepse, $35,5 \%$ foram choque séptico, $25,8 \%$ sepse grave e $19,4 \%$ sepse, sendo o foco presumido com predomínio do aparelho respiratório $(54,8 \%)$, seguido do aparelho abdominal $(25,8 \%)$ e pele/ partes moles $(16,1 \%)$. Ainda foi visto no estudo que o índice de choque, representado pela pressão arterial sistólica dividido pela frequência cardíaca na admissão, foi de 1,67 , com desvio padrão de 0,70. (Gráficos 1 e 2)

Gráfico 1. Prevalência de sepse nos pacientes internados na Unidade de Terapia Intensiva do Hospital Geral de Fortaleza.

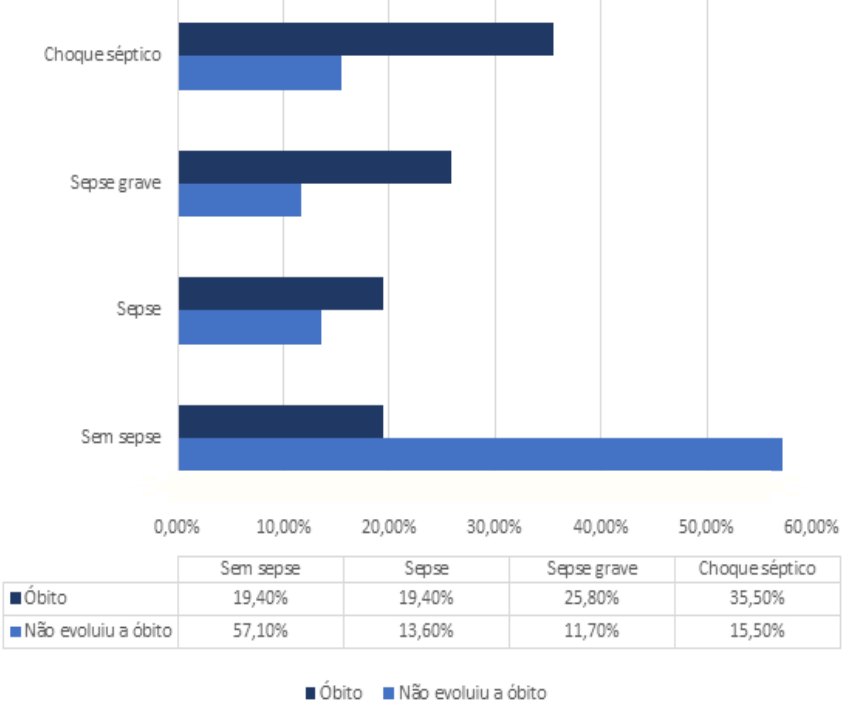

Em relação às disfunções na admissão na UTI dos pacientes que foram a óbito, $71,0 \%$ apresentava disfunção neurológica, $58,1 \%$ respiratória, $48,4 \%$ cardiocirculatória, $48,4 \%$ renal, $48,4 \%$ hematológica e $29 \%$ hepática, todos com valores de $p$ significativos, exceto a disfunção renal $(p \leq 0,025)$. As medidas de suporte mais utilizadas foram Ventilação Mecânica (77,4\%), Drogas Vasoativas (41,9\%) e Diálise (12,9\%) (Gráfico 3).

Entre as comorbidades, as principais foram: Hipertensão 
Arterial (58,1\%), Imunossupressão (25,8\%), Tabagismo (22,6\%), Etilismo (19,4\%), Diabetes Mellitus (19,4\%), Doença Arterial Coronariana (19,4\%), Insuficiência Cardíaca (12,9\%), Obesidade mórbida (12,9\%) e Acidente Vascular Cerebral prévio (12,9\%). Houve uma média de três comorbidades nos pacientes estudados.

Gráfico 2. Prevalência de focos infecciosos nos pacientes internados na Unidade de Terapia Intensiva do Hospital Geral de Fortaleza.

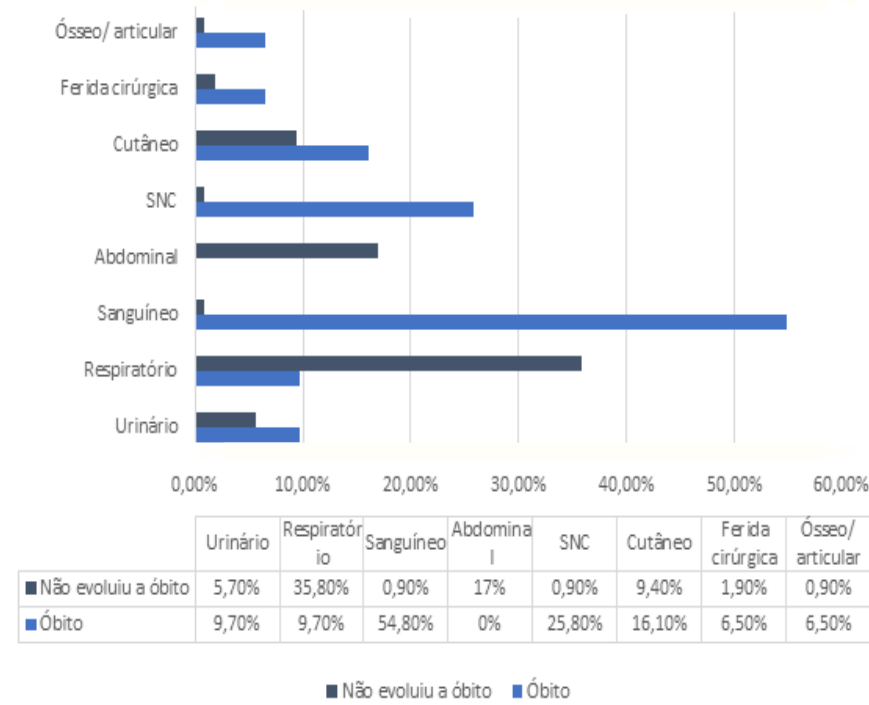

Gráfico 3. Prevalência das disfunções na admissão nos pacientes internados na Unidade de Terapia Intensiva (UTI) do Hospital Geral de Fortaleza que evoluíram a óbito.

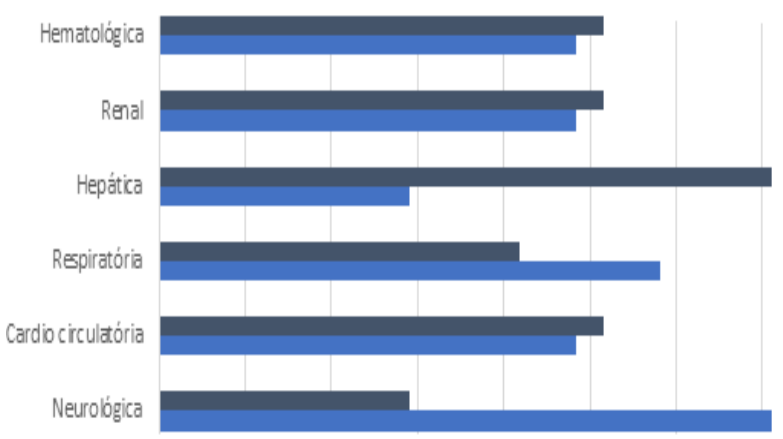

\begin{tabular}{|c|c|c|c|c|c|c|}
\hline & 10,00 & $20,00 \%$ & $30,00 \%$ & $50,00 \%$ & $60,00 \%$ & $80,00 \%$ \\
\hline & Neurob́gica & $\begin{array}{l}\text { Cardio } \\
\text { circulatória }\end{array}$ & Respiratória & Hepática & Renal & Hematológica \\
\hline - Nõ̃o presente & $29 \%$ & $51,60 \%$ & $41,90 \%$ & $71 \%$ & $51,60 \%$ & $51,60 \%$ \\
\hline aresente & $71,00 \%$ & $48,40 \%$ & $58,10 \%$ & $29 \%$ & $48,40 \%$ & $48,40 \%$ \\
\hline
\end{tabular}

- Não presente I Presente

Aplicando-se os escores de mortalidade APACHE-II e SOFA, observa-se que entre os pacientes que vieram a óbito, o APACHE-II resultou em escore médio de 22 pontos, com desvio padrão de 8 pontos, e a mortalidade prevista média foi de 55,7 $(p<0,001)$. O SOFA teve média no primeiro dia de 7 pontos $(p<0,001)$, no quinto dia de 5 pontos $(p=0,004)$ e no décimo dia de 5 pontos $(p=0,004)$. Entre as comorbidades identificadas pelo SOFA na admissão, os pacientes possuíam maior grau de comprometimento de disfunção cardiocirculatória (45,2\%), seguida de disfunção renal $(16,1 \%)$ e disfunção neurológica $(12,9 \%)$, tendo significância estatística nas disfunções cardiocirculatória e neurológica $(p<0,05)$. No quinto dia, destacava-se a disfunção cardiocirculatória $(16,1 \%)$, tendo significância estatística $(p=0,018)$, seguida de disfunção neurológica $(12,9 \%)$ e disfunção renal $(9,7 \%)$. No décimo dia, continuava o destaque para disfunção cardiocirculatória $(22,6 \%)$, tendo significância estatística $(p<0,001)$, seguida de disfunção renal (12,9\%) e disfunção neurológica $(9,7 \%)$.

\section{DISCUSSÃO}

Em nosso estudo, foi identificada a mortalidade de $24,48 \%$. Um ponto importante em avaliação de pacientes em UTI é a análise das informações sociodemográficas e epidemiológicas desses para definir estratégias qualitativas e quantitativas para melhorar o atendimento aos pacientes, especialmente na prevenção de complicações, atendimento especializado e acesso à reabilitação ${ }^{14}$.

Quanto ao sexo, percebe-se uma maior mortalidade no sexo masculino. Tal dado é explicado em virtude de maior prevalência desse gênero na UTI, devido ao baixo interesse da busca pela melhoria da saúde por parte dos homens. Quando acontece a adesão por parte deles, geralmente a gravidade da doença já está estabelecida ${ }^{14}$.

Percebe-se uma maior mortalidade em pacientes internados em UTI que se encontram na faixa etária entre 60 a 79 anos. (15) Essa informação corrobora o achado do nosso estudo em virtude de os óbitos acontecerem principalmente em pacientes em torno de 60 anos, com um desvio-padrão de 16 anos. Nesse ponto, percebe-se a importância do envelhecimento populacional associada à transição demográfica, mudando o padrão de mortes provocadas por doenças infecciosas e transmissíveis por óbitos decorrentes de doenças crônicas e degenerativas ${ }^{14}$.

Percebe-se, também, a importância da quantidade de dias internados na UTI em relação à mortalidade. Nosso estudo demonstra uma relação proporcional do número de dias de internação relacionado ao número de óbitos comparando com o tempo de 48 horas, corroborando outro estudo o qual diz que $7 \%$ evoluíram para o óbito em menos de 48 horas e $25,7 \%$ após as primeiras 48 horas $^{16}$.

Conclui-se como principal causa de óbito para os pacientes internados em UTI são os casos clínicos, fato que é corroborado por um estudo que determinou que os pacientes que foram a óbito tiveram como principal causa as Doenças do Sistema Circulatório (43\%), seguida pelas Doenças do Sistema Respiratório (21\%) e Doenças Infecciosas e Parasitárias (14\%) ${ }^{15}$

Com o envelhecimento da população, é crescente a demanda por leitos de UTI, o que pode ser causa de agilização de alta para 
enfermarias de pacientes ainda não totalmente livres de risco. (17) Um dos pontos destacados no nosso estudo direciona-se ao número de leitos restritos nos hospitais públicos atualmente, o que complica mais quando há leitos disponibilizados por ordem de mandato judicial. Diante dessa constatação, percebe-se que o Brasil está longe de atender as necessidades quanto ao número de leitos em UTI, em especial em serviços públicos ${ }^{18}$.

A hipertensão arterial sistêmica (HAS) é o principal fator de risco para AVE, estando presente em até $65 \%$ dos pacientes internados, (19) o qual corrobora nosso estudo que demostrou o Acidente Vascular Cerebral (22,6\%) como principal diagnóstico dos pacientes internados em UTI. Tal dado é explicado por doenças neurológicas ou cerebrovasculares (DCV), que são importante causa de morbimortalidade, fazendo que o paciente neurológico necessite de avaliação contínua ${ }^{20}$.

Há alta incidência de pacientes que usaram a ventilação mecânica (82\%), vasopressores (66,2\%), hemotransfusão $(44,7 \%)$ e cateter de SwanGanz $(18,8 \%)$, estando de acordo com o estudo presente em que se verificou como medidas de suporte mais utilizadas a Ventilação Mecânica (77,4\%), o uso de Drogas Vasoativas (41,9\%) e a realização de Diálise (12,9\%). ${ }^{21}$.

A sepse é a principal causa de morte nas Unidades de Terapia Intensiva (UTI) e está entre as principais causas de morte nos EUA $^{22}$. O presente estudo demonstrou que houve uma predominância de sepse de $80,6 \%$ nos pacientes que evoluíram a óbito, sendo que desse total $35,5 \%$ foram choque séptico, $25,8 \%$ sepse grave e $19,4 \%$ sepse. Nosso estudo encontra-se em concordância com o estudo SOAP, realizado em 198 UTIS Europeias, demonstrou uma mortalidade na sepse grave de $32,2 \%$ e no choque séptico de $54,1 \%{ }^{23}$. Um estudo brasileiro chamado BASES apresentou dados semelhantes ao presente estudo, divergindo apenas no sexo em que foi predominante o sexo masculino no BASES e relatando com predominância o foco séptico sendo o pulmonar ${ }^{24}$.

Em relação às disfunções orgânicas, percebe-se uma associação direta com o motivo da internação dos pacientes. A maioria das causas de internações nesta faixa etária está, de acordo com estes estudos, relacionada às doenças cardiovasculares (incluindo as cerebrovasculares) e respiratórias ${ }^{25}$, o que também foi demonstrado em nossa casuística, em que 137 pacientes ( $82 \%$ dos idosos) foram internados por AVC e/ou doenças respiratórias.

Quanto às comorbidades, o estudo presente mostrou alta prevalência de Hipertensão Arterial Sistêmica (58,1\%), Imunossupressão (25,8\%), Tabagismo (22,6\%), Etilismo (19,4\%), Diabetes Mellitus (19,4\%), Doença Arterial Coronariana (19,4\%), Insuficiência Cardíaca (12,9\%), Obesidade mórbida (12,9\%) e Acidente Vascular Cerebral prévio (12,9\%). Ressalta-se que o tabagismo, a dislipidemia e o DM constituem os fatores de risco mais críticos para a HAS, o que confirma a importância de medidas preventivas para a doença em toda a população, seja por meio de mudanças de hábitos ou pela terapêutica medicamentosa ${ }^{19,26}$.

Os escores APACHE II e SOFA apresentaram notável relação com a mortalidade, com escore médio de 22 pontos com desvio-padrão de 8 pontos e ambos com significância de $p<0,001$ no primeiro dia. Os pacientes possuíam maior grau de comprometimento de disfunção cardiocirculatória $(45,2 \%)$, seguida de disfunção renal $(16,1 \%)$ e disfunção neurológica $(12,9 \%)$, tendo significância estatística em disfunção cardiocirculatória e neurológica $(p<0,05)$. No quinto dia, destacava-se a disfunção cardiocirculatória $(16,1 \%)$, tendo significância estatística em disfunção cardiocirculatória $(p=0,018)$, seguida de disfunção neurológica $(12,9 \%)$ e disfunção renal $(9,7 \%)$. No décimo dia, continuava o destaque para disfunção cardiocirculatória $(22,6 \%)$, tendo significância estatística em disfunção cardiocirculatória ( $p<0,001)$, seguida de disfunção renal $(12,9 \%)$ e disfunção neurológica $(9,7 \%)$. A literatura é acorde em que a disfunção respiratória é mais prevalente, no entanto, as disfunções cardiovascular e neurológica correlacionaram-se com as maiores taxas de mortalidade. Além disso, o escore SOFA encontra-se maior no primeiro dia, tendendo a diminuir com a evolução do quadro do paciente ${ }^{27,28,29}$.

\section{CONCLUSÃO}

O estudo evidenciou a importância de uma análise epidemiológica quanto ao número de óbitos ocorridos em Unidades de Terapia Intensiva (UTI), destacando-se como objetivo a análise das ações de planejamento e organização de políticas e protocolos que ajudem os profissionais de saúde a abordarem os pacientes internados nesse setor e compreender as principais etiologias, disfunções e evolução quanto à idade, gênero e complicações desses pacientes. Para isso, demonstrase a necessidade de se conhecer melhor a aplicabilidade dos escores SOFA e APACHE II e as medidas que devem ser adotadas em casos de alto risco de mortalidade.

\section{REFERÊNCIAS}

1. Queiroz F, Rego D, Nobre G. Morbimortalidade na unidade de terapia intensiva de um hospital público. Rev baiana enferm. 2013 Maio-Ago; 27(2):164-171.

2. Moritz RD, Beduschi G, Machado FO. Avaliação dos óbitos ocorridos no Hospital Universitário da Universidade Federal de Santa Catarina (HU/UFSC). Rev Assoc Med Bras [Internet]. 2008 Set-Out. [acesso 2017 Mar 14]; 54(5): 390-395. Disponível em: http://www.scielo.br/scielo.php?script=sci_arttext\&pid=S010442302008000500009\&Ing=en.

3. Abrahão ALCL. A Unidade de terapia intensiva. In: Cheregatti AL, Amorim CP. Enfermagem em unidade de terapia intensiva. 2. ed. São Paulo: Martinari; 2010.
4. Oliveira ABF, Dias OM, Mello MM, Araújo S, Dragosavac D, Nucci A, et al . Fatores associados à maior mortalidade e tempo de internação prolongado em uma unidade de terapia intensiva de adultos. Rev bras ter intensiva [Internet]. 2010 Jul-Set. [acesso 2017 Mar 14]; 22(3): 250-256. Disponível em: http://www.scielo.br/scielo.php?script=sci_arttext\&pid=S0103507X2010000300006\&lng=en.

5. Garland A, Olafson K, Ramsey CD, Yogendran M, Fransoo R. Epidemiology of critically ill patients in intensive care units: a population-based observational study. Critical Care [Internet]. 2013 Set. [acesso 2017 Mar 14]; 17(5): R212. Disponível em: https://www.ncbi.nlm.nih.gov/pmc/articles/PMC4056438/. 
6. Abelha FJ, Castro MA, Landeiro NM, Neves AM, Santos CC. Mortalidade e o tempo de internação em uma unidade de terapia intensiva cirúrgica. Rev Bras Anestesiol [Internet]. 2006 Fev. [acesso 2017 Mar 15]; 56(1): 34-45. Disponível em: http://www.scielo.br/scielo.php?script=sci_arttext\&pid=S0103507X2010000300006\&lng=en.

7. Hon KL, Luk MP, Fung WM, Li CY, Yeung HL, Liu PK al. Mortality, length of stay, bloodstream and respiratory viral infections in a pediatric intensive care unit. J Crit Care [Internet]. 2017 Abr. [acesso 2017 Mar 15]; 38:57-61. Disponível em: http://www.sciencedirect.com/science/article/pii/S0883944116305482.

8. Gershengorn HB, Harrison DA, Garland A, Wilcox ME, Rowan KM, Wunsch $\mathrm{H}$. Association of Intensive Care Unit Patient-to-Intensivist Ratios With Hospital Mortality. JAMA Intern Med [Internet]. 2017 Mar. [acesso 2017 Mar 15]; 177(3): 388-96. Disponível em: http://jamanetwork.com/journals/ jamainternalmedicine/fullarticle/2598511.

9. Friedrich JO, Wilson G, Chant C. Long-term outcomes and clinical predictors of hospital mortality in very long stay intensive care unit patients: a cohort study. Crit Care [Internet]. 2006 [acesso 2017 Mar 15]; 10(2): R59. Disponível em: https://ccforum.biomedcentral.com/articles/10.1186/cc4888.

10. Laupland KB, Kirkpatrick AW, Kortbeek JB, Zuege DJ. Long-term mortality outcome associated with prolonged admission to the ICU. Chest [Internet]. 2006 Abr. [acesso 2017 Mar 15]; 129(4): 954-9. Disponível em: http://www. sciencedirect.com/science/article/pii/S0012369215388115.

11. Favarin SS, Camponogara S. Perfil dos pacientes internados na unidade de terapia intensiva adulto de um hospital universitário. Rev Enferm [Internet]. 2012 Maio-Ago. [acesso 2017 Mar 15]; 2(2): 320-9. Disponível em: https:// periodicos.ufsm.br/reufsm/article/view/5178.

12. Mares CM, Biondi RS, Sotero S, Lima AA; Almeida, KJQ; Amorim, FF. Perfil epidemiológico e preditores de mortalidade de uma unidade de terapia intensiva geral de hospital público do Distrito Federal. Com ciênc. saúde [Internet]. 2015 Maio [acesso 2017 Mar 15];26(1/2): 9-19. Disponível em: http://pesquisa.bvsalud.org/bvsms/resource/pt/mis-37657.

13. Acuña K, Costa E, Grover A, Camelo A, Santos Júnior R. Características clínicoepidemiológicas de adultos e idosos atendidos em unidade de terapia intensiva pública da Amazônia (Rio Branco, Acre). Rev bras ter intensiva [Internet]. 2007 Jul-Set. [acesso 2017 Mar 15]; 19(3):304-9. Disponível em: http://www.scielo. $\mathrm{br} / \mathrm{scielo}$.php?script=sci_arttext\&pid=S0103-507X2007000300006\&lng=en .

14. Hernández RA, Camargo BMB, Fátima PO, Giseli Z, Hernández RMJ. Características epidemiológicas e causas de óbitos em pacientes internados em terapia intensiva. Rev Bras Enferm . 2016 Mar-Abr; 69(2): 229-34. doi: http:// dx.doi.org/10.1590/0034-7167.2016690204i.

15. Otto GLG, Barbosa ADC, Francioli ALS, Costa CKF, Yamaguchi MU. Análise de óbitos na lista de espera da central de regulação de leitos de UTI na macrorregião de Maringá-PR. Enciclopédia Biosfera [Internet]. 2013 [acesso 2017 Mar 19]; 9(17): 2904-12. Disponível em: http://www.conhecer.org.br/enciclop/2013b/ CIENCIAS\%20DA\%20SAUDE/ANALISE\%20DE\%20OBITOS.pdf.

16. Feijó CAR, Bezerra ISAM, Peixoto Júnior AA, Meneses FA. Morbimortalidade do idoso internado na Unidade de Terapia Intensiva de Hospital Universitário de Fortaleza. Rev bras ter intensiva [Internet]. 2006 Set. [acesso $2017 \mathrm{Mar}$ 24]; 18(3): 263-67. Disponível em: http://www.scielo.br/scielo.php?script=sci_ arttext\&pid=S0103-507X2006000300008\&lng=en.

17. Giacomini MG, Lopes MVCA, Gandolfi JV, Lobo SMA. Choque séptico: importante causa de morte hospitalar após alta da unidade de terapia intensiva. Rev bras ter intensiva [Internet]. 2015 Mar. [acesso 2017 Mar 24]; 27(1): 51-6. Disponível em: http://www.scielo.br/scielo.php?script=sci_arttext\&pid=S0103507X2015000100051\&lng=en.

18. Hermans G, Van Mechelen H, Clerckx B, Vanhullebusch T, Mesotten D,
Wilmer A et al. Acute Outcomes and 1-Year Mortality of Intensive Care Unitacquired Weakness. A cohort study and propensity-matched analysis. Am J Respir Crit Care Med. 2014 Ago;190(4):410-20. doi: https://doi.org/10.1164/ rccm.201312-22570C.

19. Pires SL, Gagliardi RJ, Gorzoni ML. Estudo das frequências dos principais fatores de risco para acidente vascular cerebral isquêmico em idosos. Arq Neuro-Psiquiatr [Internet]. 2004 Set. [acesso 2017 Mar 24]; 62(3b): 844-851. Disponível em: http://www.scielo.br/scielo.php?script=sci_arttext\&pid=S0004$282 \times 2004000500020 \& \operatorname{lng}=$ en

20. Silva MPP, Carvalho NZ, Pires JO, Paula PH, Gomes GLO, Costa CKF et al. Causas evitáveis de internamento em unidade de terapia intensiva. Iniciação Científica - CESUMAR [Internet]. 2013 Jul.-Dez. [acesso 2017 Mar 25]; 15(2): 147-55. Disponível em: http://periodicos.unicesumar.edu.br/index.php/ iccesumar/article/viewFile/3189/2287.

21 Sales JAL Júnior, David CM, Hatum R, Souza PCSP, Japiassú A, Pinheiro CTS et al. Sepse Brasil: estudo epidemiológico da sepse em Unidades de Terapia Intensiva brasileiras. Rev bras ter intensiva [Internet]. 2006 Jan-Mar. [acesso 2017 Mar 25]; 18(1): 9-17. Disponível em: http://www.scielo.br/scielo. php?script=sci_arttext\&pid=S0103-507X2006000100003\&lng=en. http:// dx.doi.org/10.1590/S0103-507X2006000100003.

22. Martin GS, Mannino DM, Eaton S, Moss M. Epidemiology of sepsis in the United States from 1979 through 2000. N Engl J Med [Internet]. 2003 Abr. [acesso 2017 Mar 25]; 348(16): 1546-54. Disponível em: http://www.nejm.org/ doi/full/10.1056/NEJMoa022139.

23. Vincent JL, Sakr $\mathrm{Y}$, Sprung $\mathrm{CL}$, Ranieri VM, Reinhart $\mathrm{K}$, Gerlach $\mathrm{H}$ et al. Sepsis in European intensive care units: results of the SOAP study. Crit Care Med [Internet]. 2006 Fev. [acesso 2017 Mar 03]; 34(2):344-53. Disponível em: http://journals.Iww.com/ccmjournal/pages/articleviewer. aspx ?year $=2006 \&$ issue $=02000 \&$ article $=00010 \&$ type $=$ abstract

24. Silva E, Pedro MA, Sogayar AC, Mohovic T, Silva CL, Janiszewski $M$ et al. Brazilian Sepsis Epidemiological Study (BASES study). Crit Care [Internet]. 2004 Ago. [acesso 2017 Mar 25]; 8(4): R251-60. Disponível em: https://ccforum. biomedcentral.com/articles/10.1186/cc2892.

25. Oliveira PC, Tufanin AT. Perfil epidemiológico da unidade de terapia intensiva de um hospital de reabilitaação [TCC]. Goiânia (GO): Pontifícia Universidade Católica de Goiás; 2013.

26. Goulart FAA. Doenças crônicas não transmissíveis: estratégias de controle e desafios para o Sistema de Saúde [Internet]. Brasília: Organização PanAmericana da Saúde; Ministério da Saúde; 2011[acesso 2017 Mar 25]. Disponível em: https://www.nescon.medicina.ufmg.br/biblioteca/imagem/4857.pdf.

27. Pontes SPB. Avaliação da gravidade e da mortalidade de pacientes atendidos na unidade de terapia intensiva adulto do Hospital Universitário Alcides Carneiro segundo o Escore SOFA[trabalho de conclusão de curso] [Internet]. Campina Grande (PB): Universidade Estadual da Paraíba; 2012 [acesso 2017 Jun 04]. Disponível em: http://dspace.bc.uepb.edu.br:8080/ xmlui/handle/123456789/846.

28. Farias LL, Pinheiro FML Júnior, Braide ASG, Macieira CL, Araújo MVUM, Viana MCC et al. Perfil clínico e laboratorial de pacientes com sepse, sepse grave e choque séptico admitidos em uma unidade de terapia intensiva. [Internet]. Rev. Saúde Públ. 2013 Jil-Set [acesso 2017 Jun 04]; 6(3): 50-60. Disponível em: http://www.repositorio.ufc.br/handle/riufc/8345.

29. Machado RL, Nascimento DCM, Raggio LR, Amitrano DA, Salomão CS, Oliveira GMM. Análise exploratória dos fatores relacionados ao prognóstico em idosos com sepse grave e choque séptico. Rev. bras. ter. intensiva [Internet]. 2009 Mar [acesso 2017 Jun 04]; 21( 1 ): 9-17. Disponível em: http://www.scielo. $\mathrm{br} /$ scielo.php?script=sci arttext\&pid=S0103-507X2009000100002\&lng=en. http://dx.doi.org/10.1590/S0103-507X2009000100002.

\section{Como citar este artigo/How to cite this article:}

Vieira AM, Parente EA, Oliveira LS, Queiroz AL, Bezerra ISAM, Rocha EHL. Características de óbitos dos pacientes internados em uma unidade de terapia intensiva de hospital terciário. J Health Biol Sci. 2019 Jan-Mar; 7 (1):26-31. 